\title{
NOTAS NOMENCLATURALES SOBRE IRIDACEAE ${ }^{1}$
}

\author{
Adolfo Espejo Serna \\ Y \\ Ana Rosa Lopez-FerRari \\ Herbario Metropolitano \\ Departamento de Biología, C.B.S. \\ Universidad Autónoma Metropolitana \\ Unidad Iztapalapa \\ Apartado postal 55-535 \\ 09340 México, D.F. \\ e mail: aes@xanum.uam.mx
}

\section{RESUMEN}

Se aclara la situación nomenclatural de $T$. vanhouttei (Baker) Espejo \& López-Ferrari, $T$. augusta Drapiez y $T$. violacea Schiede ex Schltdl. y se proponen nuevas combinaciones en Sisyrinchium.

\section{ABSTRACT}

The nomenclatural status of $T$. vanhouttei (Baker) Espejo \& López-Ferrari, T. augusta Drapiez, and T. violacea Schiede ex Schltdl. is clarified. New combinations in Sisyrinchium are proposed.

Al adelantar los estudios correspondientes a la familia Iridaceae para la Flora del Bajío y de Regiones Adyacentes, para la Flora de Veracruz y para el listado definitivo del volumen respectivo de Las Monocotiledóneas Mexicanas, una Sinopsis Florística, nos encontramos con diversos problemas taxonómicos y/o nomenclaturales. Con el propósito de resolver algunos de ellos presentamos las siguientes notas.

1. Los nombres Tigridia vanhouttei Roezl y T. houttei Roezl fueron usados originalmente por Louis van Houtte en 1875 en una pequeña nota aparecida en el número 21 de la revista Flore des Serres et des Jardins de l'Europe. El primero de ellos aparece textualmente como "TIGRIDIA? VAN HOUTTEI Roezl" en el pie de figura de la lámina 2174 (pág. 48), en tanto que el segundo se encuentra como "TIGRIDIA HOUTTEI B. Roezl" encabezando la nota propiamente dicha (pág. 49) la cual consta de apenas nueve renglones (Apéndice 1) en los que se explica que la especie fue cultivada en Europa a

\footnotetext{
1 Trabajo parcialmente realizado con apoyo de la Comisión Nacional para el Conocimiento y Uso de la Biodiversidad.
} 
partir de semillas enviadas por B. Roezl, quien las recolectó "sur le mont Istapalapa" (Cerro de la Estrella), pero no se proporciona ninguna descripción o diagnosis de la especie. Por lo tanto y de acuerdo con los artículos 32 y 36 del Código Internacional de Nomenclatura Botánica (Greuter et al., 1994), ninguno de los dos nombres se encuentra válidamente publicado. El primero de ellos incluso aparece con un signo de interrrogación (?) intermedio. Probablemente por esta razón en el Index Kewensis el nombre que se acepta como bueno es el segundo ( $T$. houttei), aunque el más conocido y utilizado es el primero ( $T$. vanhouttei).

Klatt (1882) transfiere el epíteto al género Beatonia, pero no valida el nombre, ya que no proporciona descripción alguna. Posteriormente Baker (1892) publica válidamente el epíteto van-houttei bajo el género Hydrotaenia, dando una descripción completa basada en el material original proporcionado por van Houtte.

En el herbario de los reales jardínes botánicos de Kew $(K)$, se encuentra un pliego que en su parte izquierda superior presenta un sobre conteniendo una flor seca, así como una carta de L. van Houtte dirigida a J. G. Baker, en donde se señala: "I beg only to send ... a bulb of the so called Tigridia vanHouttei by Roezl - and I enclose the flower of the same. The leaves are ...", lo que nos permite concluir que dicho material fue el que envió van Houtte y en el cual Baker basó su descripción. Por lo tanto y de acuerdo con el artículo 9.1 del Código Internacional de Nomenclatura Botánica (Greuter et al., 1994), la flor contenida en el sobre es el holotipo de Hydrotaenia vanhouttei Baker.

En su revisión del género Tigridia para México y Centroamérica, Molseed (1970) reseña parcialmente la situación anterior. Sin embargo y sin justificarlo adecuadamente, designa un neotipo para el nombre (C. G. Pringle 4490), lo cual resulta superfluo ya que como se mencionó antes existe el holotipo. Cabe señalar que el mismo pliego en el que se encuentra el material original, tiene montado en la parte derecha un duplicado del supuesto neotipo designado por Molseed.

Dado lo anteriormente expuesto el nombre y la sinonimia correctos de esta especie son:

Tigridia vanhouttei (Baker) Espejo \& López-Ferrari, comb. nov. Basiónimo: Hydrotaenia vanhouttei Baker, Handb. Irid. 69-70. 1892, "van-Houttei". Tigridia? vanhouttei Roezl ex van Houtte, Fl. Serres 21: t. 2174. 1875, nomen nudum. Tigridia houttei Roezl ex van Houtte, FI. Serres 21: 49. 1875, nomen nudum. Beatonia houttei (Roezl ex van Houtte) Klatt, Abh. Naturf. Ges. Halle 15: 369. 1882, nomen nudum. Tipo: Flor enviada por L. van Houtte a J. G. Baker, obtenida de plantas cultivadas en Gante, Bélgica (Holotipo: $\mathrm{K}$ ! flor en el sobre de la esquina superior izquierda del pliego).

Material examinado: Distrito Federal: delegación Tláhuac, 2 km al NW de San Francisco Tlaltenango, ladera S-SE del cerro Santa Catarina, J. García 1669 (CHAP, MICH, $\mathrm{XAL}$ ); delegación Tláhuac, vertiente E del cerro de Santa Catarina, cerca de Santa Catarina, J. Rzedowski 26053 (MICH); delegación Iztapalapa, on north and east sides of Cerro de Estrella, E. Molseed 113 (MICH); delegación Iztapalpa, among ledges, Cerro de Iztapalapa "Ixtapalapa", 13.VIII.1893, C. G. Pringle 4490 (BM, F, K, M, MEXU, MO, MSC, NY, P, UC, US, VT); delegación G. A. Madero, Sierra de Guadalupe, 2 km al N de Cuautepec, S. Moreno 317 (MICH). México: $1 \mathrm{~km}$ sobre la desviación a Acozac, carretera México-Ixtapaluca, $M$. Flores C. y F. Riveros 455 (UAMIZ); municipio de Huehuetoca, Cerro Ahumada, cerca del Rancho Nuevo, 4 km al N de Huehuetoca, J. Rzedowski 28401 (MICH); 22 km al NE de 
Texcoco, sobre la carretera a Calpulalpan, J. Rzedowski 28520 (MICH); San Nicolás Tlamincas, A. Ventura A. 1362 (XAL); La Colmena, A. Ventura A. 4014 (XAL); Molino de Flores, A. Ventura A. 4062 (MICH, XAL). Querétaro: alrededores del Derramadero, J. Rzedowski 50062 (XAL). Tlaxcala: ca. $12 \mathrm{~km}$ al W de Calpulalpan, $1.4 \mathrm{~km}$ antes del límite con el estado de México, J. Ceja, A. Espejo y A. R. López Ferrari 56 (UAMIZ); municipio de Santa Cruz Tlaxcala, vicinity of San Bernabé Amaxac, E. Hernández X. 350 (MICH).

T. vanhouttei tiene una amplia distribución en la región central de México, donde se conoce de los estados de Puebla, México, Morelos, Hidalgo, Guanajuato, Querétaro, Tlaxcala y del Distrito Federal, habitando pastizales en bosques de encino, de encino-pino y de pino y matorrales xerófilos a elevaciones que van de los 2100 a los $2900 \mathrm{~m}$. Aparentemente se ve favorecida por el disturbio ya que es común hallarla también en campos de cultivo y como ruderal. La especie pertenece al subgénero Hydrotaenia sensu Molseed (1970) constituido por 18 especies, de las 29 que tiene el género (Espejo y LópezFerrari, 1996a, 1996b).

2. Durante mucho tiempo, el nombre T. violacea, publicado por Schlechtendal en 1838, se ha usado comúnmente para designar a una especie que habita a lo largo del Eje Neovolcánico Transmexicano. Sin embargo, el examen de la literatura nos permitió percatarnos de que este nombre ha sido incorrectamente utilizado, ya que existe un binomio previamente asignado a este taxon.

Drapiez (1832) describió T. augusta a partir de plantas obtenidas de semillas colectadas por Vicente Cervantes procedentes de México. La amplia descripción que acompaña a este nombre (Apéndice 2), junto con la lámina que la ilustra, no dejan lugar a dudas sobre la identidad de la misma con T. violacea. El Dr. Paul Bamps, curador del herbario de Bruselas, tuvo la amabilidad de comunicarnos que los registros del jardín de la Real Sociedad de Horticultura indican que en efecto T. augusta fue cultivada ahí en 1832, pero que desafortunadamente no se conservó material herborizado. En consecuencia la lámina que acompaña al protólogo puede considerarse como tipo del nombre.

Por lo tanto el nombre y la sinonimia correctos para esta especie son:

Tigridia augusta Drapiez, in van Geel, Sert. Botanicum 4: t. 131 1832. Tipo: De plantas cultivadas en Bruselas, a partir de semillas colectadas por V. Cervantes procedentes de México. Lectotipo (aquí designado); lámina 131 de Sertum Botanicum 4. 1832.

Tigridia violacea Schiede ex Schltdl., Allg. Gartenzeitung 6: 233. 1838. Beatonia purpurea Herb., Bot. Mag. 66: t. 3779. 1840. Tipo: Floruit apud dom. T. Harris ex ditione Mexicana allata, curante hortulano perito D. Beaton. Vivam. inspexi. (Neotipo (Molseed, 1970): México, Tultenango, 13.VII.1901, C. Pringle s. n. (VT!)) syn. nov.

Material examinado: Michoacán: municipio de Zinapécuaro, Llano Largo, cerca de Los Azufres, H. Díaz Barriga 3930 (IEB, UAMIZ); municipio de Morelia, $1 \mathrm{~km}$ después de la desviación a San José de Coapa, sobre la carretera Pátzcuaro-Morelia, M. Flores, A. Espejo, A. R. López Ferrari y H. Díaz Barriga 442 (UAMIZ); on highway 10 just east of El Fresno, below turnoff from highway 15, E. Molseed $440(\mathrm{MICH})$. Puebla?: $18 \mathrm{~km}$ en route to Morelia (from Puebla), H. M. Chute M-171 (MICH). México: alrededores de la presa de 
La Concepción, 8 km al WSW de Tepotzotlán, J. Rzedowski 16788 (MICH); municipio de Tepozotlán, alrededores de la presa de La Concepción, J. Rzedowski 38404 (XAL). Jalisco: municipio de Tapalpa, $19 \mathrm{~km}$ al SW de la desviación a Lagunillas, de la carretera TapalpaGuadalajara, rancho abandonado cerca de El Salto, Río Jiquilpan, E. Lott et al. 369 (MICH); municipio de Autlán, $5 \mathrm{~km}$ al S de Ahuacapan, E. Lott y J. Magallanes 451 (MICH).

T. augusta se distribuye en la parte central de México en los estados de Guanajuato, Jalisco, México, Michoacán, Puebla y Zacatecas. Habita preferentemente en llanos y lugares soleados y expuestos, entre los 1900 y los $3000 \mathrm{~m}$ de altitud. La especie pertenece al subgénero Tigridia sensu Molseed (1970) constituido por 11 especies, de las 29 que tiene el género (Espejo y López-Ferrari, 1996a, 1996b).

Cabe mencionar aquí que Sisyrinchium cervantesii fue descrito por E. Meyer en la obra de C. Presl, Reliquiae Haenkeanae (pág. 117, 1827). El tipo de dicho nombre fue aparentemente recolectado por Vicente Cervantes, ya que en el protólogo se indica: "Hab. in Mexico. Herbarium Cervantesii". Las colecciones que se conservan en el país de este botánico mexicano se encuentran depositadas en el Herbario Nacional (MEXU). Infortunadamente, después de una revisión cuidadosa de las mismas no pudimos encontrar ningún ejemplar asignable a dicho taxon. Indagaciones en los herbarios de Praga (PR y PRC) a través de la Dra. Blanka Skočdopolová, nos llevaron a concluir que en tales museos no existe tampoco material herborizado de $S$. cervantesii.

Klatt (1863) tuvo la oportunidad de revisar el espécimen original depositado en el herbario Haenkeano (PR y/o PRC) y señaló que $S$. cervantesii corresponde en realidad a Beatonia purpurea Herb., nombre que diversos autores (Baker, 1892, 1894; Hemsley, 1884; Klatt, 1861-62; Molseed, 1970) han considerado como sinónimo de Tigridia violacea Schiede ex Schltdl. Si esto es cierto, el epíteto cervantesii tendría prioridad sobre el de augusta.

Por otra parte, McVaugh (1989) indicó que Sisyrinchium cervantesii Meyer podría ser un nombre anterior aplicable a la especie que se ha identificado como Sisyrinchium scabrum Schltdl. \& Cham., sin embargo basó su aseveración en la breve descripción de Meyer y comentó que no vio el tipo. En virtud de que hasta donde sabemos, probablemente no existe el material original y de que no es posible determinar la correcta aplicación del nombre, preferimos rechazarlo considerándolo nomen confusum.

3. Bicknell (1900) describió varias especies nuevas de Hydastylus a partir de material proveniente de México. Actualmente (Rudall et al., 1986; Goldblatt et al., 1989, 1990) Hydastylus se considera como parte del subgénero Echthronema (Herb.) Goldblatt de Sisyrinchium, por lo que se hacen necesarias las siguientes combinaciones:

Sisyrinchium parvum (E. P. Bicknell) Espejo \& López-Ferrari, comb. nov. Basónimo: Hydastylus parvus E. P. Bicknell, Bull. Torrey Bot. Club 27: 384. 1900. Tipo: Coahuila, low valley near Saltillo, 22.III.1847, J. Gregg 340 (MO; NY!).

Sisyrinchium serrulatum (E. P. Bicknell) Espejo \& López-Ferrari, comb. nov. Basónimo: Hydastylus serrulatus E. P. Bicknell, Bull. Torrey Bot. Club 27: 385-386. 1900. Tipo: Veracruz, Orizaba, in the mountains, M. Botteri 1856 (US; NY!). 
Sisyrinchium translucens (E. P. Bicknell) Espejo \& López-Ferrari, comb. nov. Basónimo: Hydastylus translucens E. P. Bicknell, Bull. Torrey Bot. Club 27: 386-387. 1900. Tipo: Baja California Sur, Sierra de la Laguna, 24.I, T. Brandegee (CAS!).

\section{AGRADECIMIENTOS}

Queremos agradecer a los Dres. Rzedowski la revisión crítica del presente manuscrito, así como sus valiosas observaciones y sugerencias. La Dra. Blanka Skočdopolová del Herbario de Praga, el Dr. Paul Bamps del Herbario de Bruselas y el Dr. Simon Owens del Herbario de Kew, amablemente nos proporcionaron fotografías e información del material tipo depositado en sus respectivas colecciones. En especial queremos hacer patente nuestro agradecimiento a la Dra. Lourdes Rico Arce del herbario de Kew, por su invaluable ayuda en la revisión física del material y por el envío de fotocopias y dibujos. Finalmente deseamos agradecer a los curadores de los herbarios por las facilidades proporcionadas para la consulta del material.

\section{LITERATURA CITADA}

Baker, J. G. 1892. Handbook of the Irideae. G. Bell \& Sons. Londres. 247 pp.

Baker, J. G. 1894. Tigridia violacea. Bot. Mag. t. 7356.

Bicknell, E. P. 1900. Studies in Sisyrinchium-VIII: Sisyrinchium californicum and related species of the neglected genus Hydastylus. Bull. Torrey Bot. Club 27: 373-387.

Drapiez, P. A. 1832. Tigridia augusta In: van Geel, Sert. Botanicum 4: t. 131.

Espejo, A. y A. R. López Ferrari. 1996a. Comentarios florístico-ecológicos sobre las iridáceas mexicanas. Acta Bot. Mex. 34: 25-47.

Espejo, A. y López Ferrari, A. R. 1996b. Las monocotiledóneas mexicanas, una sinopsis florística. 1. Lista de referencia. Parte VI. Dioscoreaceae a Nolinaceae. Consejo Nacional de la Flora de México, A.C., Universidad Autónoma Metropolitana y Comisión Nacional para el Conocimiento y Uso de la Biodiversidad. México, D. F. 120 pp.

Goldblatt, P., J. E. Henrich y R. C. Keating. 1989. Seed morphology of Sisyrinchium (IridaceaeSisyrinchieae) and its allies. Ann. Missouri Bot. Gard. 76: 1109-1117.

Goldblatt, P., J. E. Henrich y J. E. Henrich. 1990. The genera of the Sisyrinchium alliance (Iridaceae: Iridoideae): Phylogeny and relationships. Syst. Bot. 15: 497-510.

Greuter, W., H. M. Burdet, W. G. Chaloner, V. Demoulin, R. Grolle, D. L. Hawks-Worth, D. H. Nicolson, P. C. Silva, F. A. Stafleu, E. G. Voss y J. McNeill (eds.) 1994. International Code of Botanical Nomencalture. Regnum Veg. 118. 389 pp.

Hemsley, W. B. 1882-1886. Botany. In: Godman F. D. y O. Salvin (eds.). Biologia Centrali Americana. Vol 3: 1-711. R. H. Porter y Dulau \& Co. Londres.

Klatt, F. W. 1861-1862. Specimen e familia Iridearum. Linnaea 31: 533-570.

Klatt, F. W. 1863. Fortsetzung der Bestimmungen von Irideen. Linnaea 32: 283-288.

Klatt, F. W. 1882. Ergänzungen und Berichtigungen zu Baker's Systema Iridacearum. Abh. Naturf. Ges. Halle 15: 335-404.

McVaugh, R. 1989. Iridaceae. Flora Novo-galiciana 15: 294-347.

Molseed, E. 1970. The genus Tigridia (Iridaceae) of Mexico and Central America. Univ. Calif. Publ. Bot. 54: 1-127. 
Presl, C. 1827. Reliquiae Haenkeanae seu descriptiones et icones plantarum. Vol. 1: 1-356.

Rudall, P., A. Y. Kenton y T. J. Lawrence. 1986. An anatomical and chromosomal investigation of Sisyrinchium and allied genera. Bot. Gaz. (Crawfordsville) 147: 466-477.

van Houtte, L. 1875. Tigridia houttei B. Roezl. Irideae. Fl. Serres 21: 49, t. 2174.

\section{APENDICE 1}

2174

TIGRIDIA HOUTTEI B. RøEZL.

Irideæ

Levée de graines que nous a envoyée du Mexique M. B. Rœzl, qui les avait recueillies sur le mont Istapalapa, cette Tigridie s'élève, dans nos cultures, à un pied de hauteur et ses fleures se succèdent en juillet.
Nous en gardons les bulbes à l'état sec, de septembre en mars, à l'instar des autres Tigridies. 
Espejo y López-Ferrari: Notas Nomenclaturales sobre Iridaceae

APENDICE 2

TIGRIDIA AUGUSTA

TIGRIDIE AUGUSTA

\section{DIFFERENTIA SPECIFICA}

TIGRIDIA AUGUSTA: caule simplici, erecto; foliis ensiformibus, striatis, plicatis, flabellatis; corolla violacea: petalis exterioribus triplo majoribus: limbo plano, brevi reflexo.

\section{SYNONYMIA}

TIGRIDIA AUGUSTA. Drapiez in Herb. Soc. Horticult. Bruxellensis.

PARMI différentes graines dont se composait un envoi fait à la Société royale d'horticulture de Bruxelles, par don N. Cervantes, professeur de botanique et directeur du jardin des plantes à l'Université de Mexico, se trouvait un très-petit paquet étiqueté Ferraria $S$. N.; les graines qu'il renfermait ayant été semées avec tous les soins convenables, elles ont procuré la satisfaction d'en voir lever la majeure partie; et après la troisième année de semis (juillet 1832), les bulbes qui en sont resultés, ont produit de trés-jolies fleurs que l'on a reconnues pour appartenir non point au genre Ferraria, comme l'on s'y attendait, mais bien au genre Tigridia; c'est, en conséquence, la troisième espèce de celui-ci que nous voyons fleurir en Europe. Puissions-nous trouver, en continuant à cultiver cette nouvelle venue, la même complaisance que nous observons au retour de chaque été, dans la Tigridie à grandes fleurs, sans contredit l'une des plus brillantes parures de nos parterres! L'heureux mélange de ces duex plantes, rivalisant d'éclat et d'élégance, sera pour le véritable amateur un renouvellement d'extase et d'admiration. La Tigridie augusta, quoique plus éphémère encore que sa devancière dans nos climats, paraît neanmoins jouir de la précieuse qualité de n'être pas très-avare de ses fleurs; celles-ci pourront même se succéder pendant assez long-temps, et presque sans interruption, lorsque les bulbes se trouveront réunis et groupés comme on le fait assez généralement pour les Tigridies à grandes fleurs.

Son bulbe est fort allongé: il a douze à quatorze lignes sur quatre à cinq de diamètre; il est composé de tuniques écailleuses, blanches, revêtues d'une pellicule fauve-rougeâtre; il est termoné inférieurement par une racine cylindrique, blanchâtre, contournée, épaisse de deux lignes environ, à sa base où elle est entourée de fibres capillaires noirâtres. De sa partie supérieure s'élèvent trois feuilles ensiformes, à pétiole engaînant, striées et plissées en éventail dans tout leur longueur qui est de quinze pouces sur quatre lignes de largeur, pointues et d'un vert gai. La tige qu'elles entourent est presque anguleuse, haut de dix à douze pouces et terminée par une spathe membraneuse, à deux folioles. Les fleurs, au nombre de trois à six, sortent successivement de cette spathe. La corolle est composée de six pétales dont trois extérieurs, très-grands et trois intérieurs, beaucoup plus petits: les premiers sont concaves à leur base qui n'occupe que le tiers de l'étendue; dans cette partie le fond de la couleur est blanchâtre, parsemé de petites taches irrégulières, d'un bleu violet; plus haut le blanc prend une nuance de jaune, et les taches se caractérisent davantage en se rapprochant, pour la forme, de celles qui ornent la robe de Léopard: leur teinte est aussi beaucoup plus foncée; le limbe est brusquement distinct de l'onglet par un pli qui le renverse angulairement; il est d'une nuance violette, uniforme, 
seulement un peu plus obscure vers le pli. Ce limbe est presque plane, ovalaire, avec les bords faiblement sinueux et le sommet terminé par une petite pointe aiguë, souvant renversée; en dessous, la couleur de l'onglet est le blanc verdâtre: celle du limbe le violet pâle. Dans les pétales intérieurs, le limbe et l'onglet quoique également renversés ou inclinés, ont l'un et l'autre la même étendue et une concavité semblable; on remarque un peu au-dessous du pli que forment ces deux parties, par leur abaissement en sens opposés, un autre repli très-voisin de la base où il dessine de chaque côté une sorte de dent; les couleurs et les dispositions des taches sont à peu près les mêmes que dans les pétales extérieurs, à l'exception du jaune qui est remplacé par du violet, ce qui fait paraître le fond plus obscur; la pointe terminale est plus allongée. Les filaments des trois étamines sont soudés ensemble, et forment autour du pistil un tube ou une colonne d'un blanc satiné; les anthères sont supportées par ces filaments et semblent en être la continuation; elles sont régulièrement repliées et étalées, s'ouvrant en dessous, chacune en deux loges renfermant un pollen brun dont les masses bordent inférieurement les côtés de l'anthère. Au milieu du tube se trouve le style qui s'élève d'un ovaire trigone, placé sous la corolle; il est terminé par trois stigmates étalés au-dessus des anthères et profondément divisés, de manière que chacun des lobes se dirige vers un des côtés d'une anthère. La capsule est cylindrique, triangulaire, déhiscente par le sommet qui représente une calotte formée de trois pièces soudées; trois loges séparées par autant de valves, renferment chacune cinq ou six semences arrondies, réniformes, d'un brun rougeâtre.

Jusqu'ici cette espèce a été cultivée en serre tempérée, dans un mélange de trois parties de terreau de bruyère et d'une de sable. Les fleurs obtenues, étant les premières, n'avaient point toute l'étendue que l'on peut espérer d'une culture suivie et de l'acclimatation. Tout porte à croire que l'on pourra facilement habituer les bulbes à la pleine terre. 